


\section{About IJMA [last updated October, $\left.1^{\text {st }}, 2021\right]$}

$\checkmark$ International Journal of Medical Arts is the Official Journal of the Damietta Faculty of Medicine, AlAzhar University, Egypt

$\checkmark$ It is an International, Open Access, Double-blind, Peer-reviewed Journal

$\checkmark$ Published four times a year

$\checkmark$ The First Issue was published in July 2019

$\checkmark$ Published under the following license: Creative Commons Attribution-ShareAlike 4.0 International Public License (CC BY-SA 4.0). It had updated from the Creative Commons license [CC BY] in volume 2, Issue 4, October 2020 About IJMA

$\checkmark$ The Egyptian Knowledge Bank hosts the web site of IJMA

$\checkmark$ The Egyptian Knowledge Bank supports IJMA

$\checkmark$ IJMA follows the regulations of the International Committee of Medical Journal Editors

$\checkmark$ IJMA is indexed in the "Directory of Open Access Journals" [15 January 2021].

$\checkmark$ IJMA is indexed in J-Gate [29-6-2021]

$\checkmark$ IJMA is a member of the International Society of Managing and Technical Editors

$\checkmark$ Listed in "Index Copernicus", "Publons", "Academic resource index [ResearchBib]" "Electronics journal library", "Eurasian Scientific Journal Index", "WorldCat" Superstar Journal Database, and "Citefactor"

$\checkmark$ IJMA introduced to the search engine [BASE] through DOAJ

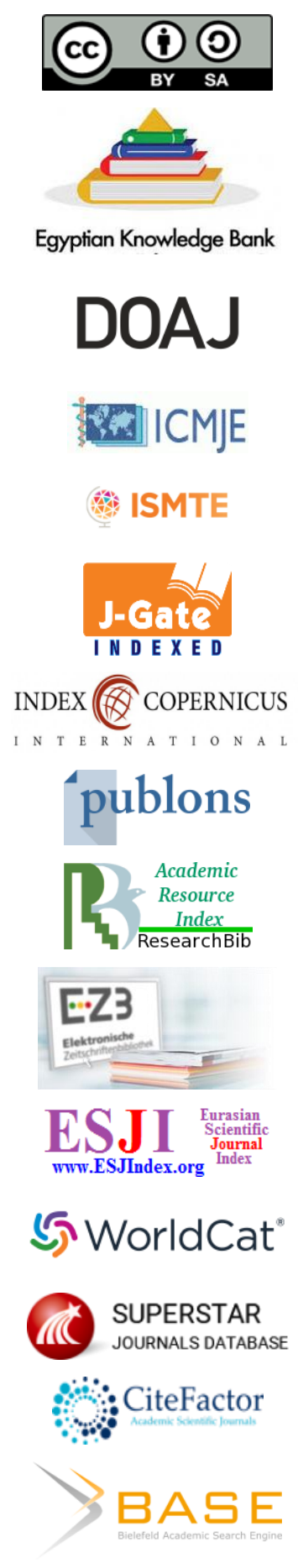




Available online at Journal Website
https://ijma.journals.ekb.eg/
Main subject [Obstetrics and Gynecology]

Original Article

\title{
Laparoscopic Hysterectomy with Prior Uterine Artery Ligation versus Conventional Laparoscopic Hysterectomy
}

\author{
Usama Mohamed Abo-Hashem [1], Rashed Mohamed Rashed ${ }^{[1]}$, Walaa Mohammed El-Bassioune ${ }^{[1]}$, Hossam \\ Abdelmageed Abdou [2]
}

1 Department of Obstetrics and Gynecology, Damietta Faculty of Medicine, Al-Azhar University, Egypt

2 Department of Obstetrics and Gynecology, Faculty of Medicine, Helwan University, Egypt

Corresponding author: Usama Mohamed Abo-Hashem

Email: pro.usa2008@gmail.com

Submission date: August 16, 2021; Acceptance date: September 16, 2021

DOI: 10.21608/IJMA.2021.91017.1352

DOAJ

\section{ABSTRACT}

Background: Hysterectomy is one of the most commonly widespread gynecologic surgeries. Total Laparoscopic Hysterectomy (TLH) being a minimally invasive surgical technique has become the new trend.

Aim of the work: The study aims at comparing TLH with previous ligation of the uterine artery at its origin, with conventional TLH regarding operative time, bleeding, intra-operative complications and postoperative follow up.

Patients and Methods: Among 60 women, who are planned for TLH, 30 women experienced TLH with previous ligation of the uterine artery (group A) and the other 30 women underwent conventional TLH (group B).

Results: The operative time of TLH in group A was significantly less than that in group B. Both blood loss and post-operative hospital stay in group $A$ were less than those in group $B$, and there were no statistically significant differences between both groups. No major intra-operative or post-operative complications occurred in both groups.

Conclusion: Total Laparoscopic Hysterectomy with Prior Uterine Artery Ligation at its origin is safe effective procedure and reducing the duration of surgery.

Keywords: Hysterectomy; Laparoscopy; Laparoscopic Hysterectomy; Uterine Artery Ligation

This is an open-access article registered under the Creative Commons, ShareAlike 4.0 International license [CC BY-SA 4.0] [https://creativecommons.org/licenses/by-sa/4.0/legalcode.

Citation: Abo-Hashem UM, Rashed RM, El-Bassioune WM, Abdou HA. Laparoscopic Hysterectomy with Prior Uterine Artery Ligation versus Conventional Laparoscopic Hysterectomy. IJMA 2021; 3 [4] October-December: 1879-1883 [DOI: 10.21608/IJMA.2021.91017.1352].

${ }^{*}$ Main subject and any subcategories have been classified according to the research topic 


\section{INTRODUCTION}

Hysterectomy is one of the most usually performed surgeries, its second common obstetrics and gynecological surgery after caesarean section, with nearly 500,000 in the United States alone ${ }^{[1]}$.

When choosing between Vaginal Hysterectomy $(\mathrm{VH})$, Abdominal Hysterectomy (AH), and Laparoscopic Hysterectomy (LH) methods, it will depend on the surgeon experience, operative indication, size and weight of the uterus, and previous abdominal operations ${ }^{[2]}$.

Much uterine pathology such as fibroids, premalignant and malignant gynecologic disorders involving the uterus can be treated using Laparoscopic Hysterectomy, which is a minimally-invasive surgical technique ${ }^{[3]}$.

\section{AIM OF THE WORK}

The main objective of the present study was to make a comparison between Total Laparoscopic Hysterectomy preceded by ligation of the uterine artery, at its arterial origin, and the conventional Total Laparoscopic Hysterectomy, as regard bleeding, operative duration, intra-operative complications, and follow up.

\section{PATIENTS AND METHODS}

This prospective cohort study was conducted at the Department of Obstetrics and Gynecology, Al-Azhar University Hospital (New Damietta) during the period between December 2017 and September 2019.

\section{Inclusion criteria}

Age > 40 years, non-malignant uterine pathologies including fibroids, endometrial hyperplasia, dysfunctional uterine bleeding failed to respond to medical treatment and pelvic prolapse. Uterine size: pelvi-abdominal swelling less than 16 weeks.

\section{Exclusion criteria}

Age $<40$ years, gynecologic malignancy, presence of contraindications to laparoscopy, like any medical condition worsened by pneumoperitoneum or the Trendelenburg position.

The study included sixty women recruited and assessed by full history and clinical examination. Trans-vaginal ultrasonography and routine preoperative laboratory investigation were done. All women fasted for a period of 8 hours before surgery.
Women were divided into 2 groups; group "A" that included 30 women who experienced ligation of the uterine artery at its origin before going on to TLH and group "B" that included 30 women who underwent Total Laparoscopic Hysterectomy.

After being subjected to general anesthesia, each patient was positioned in the modified lithotomy position and a urinary bladder catheter was inserted. A Veress needle was used for insertion at the level of umbilicus or the supraumbilical level. The patient's abdomen was insufflated using carbon dioxide $20 \mathrm{mmHg}$ initially then maintained at 15 $\mathrm{mmHg}$. A trocar about $10 \mathrm{~mm}$ in size was blindly inserted followed by introduction of $10 \mathrm{~mm}$ telescope via this port, then two additional $5 \mathrm{~mm}$ ports were introduced. The abdomen was examined before beginning surgery. Size and course of uterus, adnexa, as well as the presence of myomas were visualized. The uterus was manipulated using the vaginal manipulator.

\section{Surgical procedures}

For women in Group "A" who experienced ligation of the uterine artery at its origin before going on to $T L H$, we follow these steps:

Step 1: Dissection of the uterine artery was carried out through the lateral approach in which dissection starts from the anterior leaf of the broad ligament. The triangle bounded with the external iliac artery, round ligament, and the infundibulo-pelvic ligament was explored. Then dissection of the areolar space was performed to identify the ureter and the beginning of the uterine artery originating from the internal iliac artery. The Hem-o-lok clips were used to ligate the uterine artery after its dissection from surrounding structures in both sides.

Step 2: The advanced bipolar diathermy (LigaSur) utilized for both the round and utero-ovarian ligament, if conserved the ovary but if ovary not conserved, the infundibulo-pelvic ligament was coagulated and cute.

Step3: Opening of the broad ligament, and exposure of uterine vessels.

Step 4: Opening of the bladder flap to expose the lower uterine segment and push the bladder away for the cervical dissection.

Step 5: Opening of the posterior peritoneum at the lower uterine segment level and the uterine vessels coagulated and cute by (LigaSur).

Step 6: Repeat step 2 in another side, with getting the 
round, infundibulo-pelvic or utero-ovarian ligament.

Step 7: The uterine vasculature was separated on the contralateral side and coagulated by advanced bipolar cautery then the vessels were moved laterally on top of cervical cup, away from the vagina, leaving about 10 or $2 \mathrm{~cm}$ cuff for the purpose of dissection.

Step 8: Dissection of the cervix from the vagina using monopolar hook. Then uterus with its cervix was delivered through the vagina, after that the vault was stitched using delayed absorbable number 1 vicryl suture. Finally, port site incisions were closed.

For women in Group "B" who underwent conventional Total Laparoscopic Hysterectomy, we follow these steps:

Step 1: The advanced bipolar diathermy (LigaSur) utilized for the round and utero-ovarian ligament, if conserved the ovary but if ovary not conserved, the infundibulo-pelvic ligament was coagulated and cute.

Step2: Opening of the broad ligament, and exposure of uterine vessels.

Step 3: Opening of the bladder flap to expose the lower uterine segment and push the bladder away for cervical dissection.

Step 4: Opening of the posterior peritoneum at the lower uterine segment level and the uterine vessels coagulated and cute by (LigaSur).

Step 5: Repeat step 2 in another side, with getting the round, infundibulo-pelvic or utero-ovarian ligament.

Step 6: The uterine vasculature was separated on the contralateral side and coagulated by advanced bipolar cautery, and then the vessels were moved laterally on top of cervical cup, away from the vagina, leaving about 1 or $2 \mathrm{~cm}$ cuff for the purpose of dissection.

Step 7: Dissection of the cervix from the vagina using monopolar hook. Then uterus with its cervix was delivered through the vagina, after that the vault was stitched using delayed absorbable number 1 vicryl suture. Finally, port site incisions were closed.

The suction apparatus was used to calculate total blood loss. Irrigation was avoided through the operation till calculation of total blood loss. Normal saline was applied for peritoneal lavage. The urinary catheter was detached 6 hours later before starting liquid diet on recovery of peristalsis. The patient has been discharged within same day or next morning and was asked for follow-up 7 days later.

\section{Ethical considerations}

Approval has been obtained from the Ethics Local Research Committee Approval number: 26112019. Written informed consent was obtained from each patient before being enrolled in the present study.

\section{Statistical analysis}

Data of the present study were statistically analyzed using the Statistical Package for the Social Sciences, "SPSS" version 25, SPSS Inc., and Chicago, IL, USA). For continuous variables, data were presented as (mean $\pm S D$ ), whereas for categorical variables data were presented as frequency and percentage. Comparisons were carried out using Pearson Chi-square test for categorical variable and the unpaired Student's t-test for continuous variables. All statistical tests were carried out using a significance level of $95 \%$. P-values $>0.05$ were considered statistically significant.

\section{RESULTS}

The study included 60 women; all of them fulfilled the inclusion criteria. Regarding the demographic data, no significant differences in age, BMl, obstetric, surgical and medical history between women of; (Group A) underwent Uterine Artery Ligation by Hem-o-lok clips at its origin before undergoing Total Laparoscopic Hysterectomy and (Group B) patients underwent conventional Total Laparoscopic Hysterectomy [Table 1].

According to indication of Laparoscopic Hysterectomy it was found to fall in one of the following four categories: fibroid manifested with pain or bleeding, adenomyosis manifested with pain or bleeding, post-menopausal bleeding or endometrial hyperplasia manifested with vaginal bleeding. The results revealed no significant differences between both groups " $A$ " and "B", regarding these indications [Table 2].

According to the operative time, there was a significant difference between both groups $(P<0.001)$. But according to blood loss and post-operative hospital stay, there were no statistically significant differences between both groups $(P=$ 0.486 and 0.452 respectively) and no major intra-operative or post-operative complications occurred in both groups [Table 3]. 
Table [1]: Age, BMI, obstetric, surgical \& medical history of women enrolled in this study

\begin{tabular}{lccc} 
& $\begin{array}{c}\text { TLH with Uterine Artery Ligation at } \\
\text { its origin Group }(\mathrm{n}=30)\end{array}$ & $\begin{array}{c}\text { Conventional Total Lap. } \\
\text { Hysterectomy Group }(\mathrm{n}=30)\end{array}$ & p-value \\
Age (year) & $45.77 \pm 4.99$ & $48.4 \pm 5.36$ & 0.054 \\
BMI & $29.48 \pm 6.13$ & $31.47 \pm 6.79$ & 0.240 \\
Previous Birth & $30(100 \%)$ & $27(95 \%)$ & 0.076 \\
No Previous Birth & $0(0 \%)$ & $3(5 \%)$ & \\
Previous CS & $12(40 \%)$ & $9(30 \%)$ & 0.417 \\
No Previous CS & $18(60 \%)$ & $21(70 \%)$ & 0.152 \\
Previous Surgery & $11(36.7 \%)$ & $6(20 \%)$ & \multirow{2}{*}{0.436} \\
No Previous Surgery & $19(63.3 \%)$ & $24(80 \%)$ & \\
Medical Disorders & $12(40 \%)$ & $15(50 \%)$ & \\
No Medical Disorders & $18(60 \%)$ & $15(50 \%)$ & \\
\hline
\end{tabular}

Table [2]: Indication of laparoscopic hysterectomy

\begin{tabular}{|l|c|c|c|}
\hline & TLH with Uterine Artery Ligation & Conventional Total Lap. & -value \\
& at its origin Group $(\mathbf{n}=30)$ & $10(33.33 \%)$ & \\
\hline Fibroid & $10(33.33 \%)$ & $8(26.67 \%)$ & \\
Adenomyosis & $10(33.33 \%)$ & $7(23.33 \%)$ & 0.907 \\
Post-menopausal bleeding & $5(16.67 \%)$ & $5(16.67 \%)$ & \\
Endometrial hyperplasia/bleeding & $5(16.67 \%)$ & & \\
\hline
\end{tabular}

Table [3]: Operative time, blood loss and post-operative hospital stay

\begin{tabular}{lccc} 
& $\begin{array}{c}\text { TLH with Uterine Artery Ligation } \\
\text { at its origin Group ( } \mathrm{n}=30)\end{array}$ & $\begin{array}{c}\text { Conventional Total Lap. } \\
\text { Hysterectomy Group ( } \mathrm{n}=30)\end{array}$ & $\mathrm{p}$-value \\
Time (min.) & $48.93 \pm 8.3$ & $58.83 \pm 9.26$ & $<\mathbf{0 . 0 0 1}^{*}$ \\
Blood loss (ml) & $97.67 \pm 34.31$ & $104.33 \pm 39.19$ & 0.486 \\
Hospital stays (hours) & $14.93 \pm 5.14$ & $16 \pm 5.75$ & 0.452 \\
\hline
\end{tabular}

\section{DISCUSSION}

Hysterectomy is one of the most usually performed surgeries, with nearly 500,000 in the United States alone, $87 \%$ of which were done for benign uterine pathology [1]. When choosing between vaginal hysterectomy, abdominal hysterectomy and laparoscopic hysterectomy, it will depend on the experience of the surgeon, surgical indication, uterine size, weight, and previous abdominal surgery [2].

Laparoscopic hysterectomy is a minimally invasive surgical technique for that can be used for treatment of abnormal uterine bleeding, pelvic pain, uterine fibroids, premalignant and malignant gynecologic conditions affecting the uterus ${ }^{[3]}$.

Therefore, we carried this prospective study on sixty patients recruited from those attending the outpatient gynecologic clinic Al-Azhar University Hospital (New Damietta), who were candidate for hysterectomy for benign gynecological disorders to compare the operative time, intraoperative blood loss, time of hospital stay, intra-operative and post-operative complication between two groups.

Our study where 30 patients underwent Uterine Artery
Ligation by Hem-o-lok clips at its origin before undergoing Total Laparoscopic Hysterectomy and (Group B where 30 patients underwent conventional Total Laparoscopic Hysterectomy.

This present study shows no significant difference between both groups according to age, BMI, parity, medical and surgical history, it also shows no significant difference between both groups according to cause of Hysterectomy, but according to time of surgery, we found significant difference between both groups; the mean time of surgery in the 1st group was $(48.93 \pm 8.3)$ minutes compared to $(58.83 \pm 9.26)$ minutes in the 2 nd group with $p$ value $<0.001$.

Our study is consistent with Sinha et al. [4]; a study conducted on two groups of patients, during Total Laparoscopic Hysterectomy "TLH". Group "1" (patients with ligated uterine arteries on starting TLH), and group "2" (patients with sutures achieved after corneal pedicles). Regarding the duration of operation, the mean time of surgery was 60 minutes in group 1 and the mean time of surgery was 70 minutes in group 2 with significant reduction in time of surgery of earlier Uterine Artery Ligation at the beginning of TLH. 
Also, this present study is consistent with Poojari et al. [5] a study of two groups; where group A underwent conventional TLH and group $B$ underwent TLH with earlier Uterine Artery Ligation at its origin; In group $A$, the mean time of surgery was 71 minutes, while in group $B$, the mean time of surgery was 60 minutes with a statistically significant difference between both groups.

Our study also is consistent with Kale et al. ${ }^{[6]}$ a study of two groups; one group underwent TLH in $(99.16 \pm 7.01)$ minutes and another group underwent TLH with Uterine Artery Ligation at the beginning in $(63.16 \pm 7.16)$ minutes with significant difference between both groups.

On other hand our study results differ from Pan et al. [7]; which was a study of TLH versus TLH with coagulation of uterine artery at its origin. TLH mean time of surgery was 95 minutes and TLH with coagulation uterine artery at its origin mean time of surgery was 97 minutes with no significant difference between both groups which differs from our study results.

In our study, there was no significant difference between both groups according to blood loss; in 1st group, the mean blood loss was $(97.67 \pm 34.31) \mathrm{ml}$ when compared to $(104.33 \pm 39.19) \mathrm{ml}$ in 2 nd group.

Pan et al. ${ }^{[7]}$ study is consistent with our study which found no significant difference of blood loss in both groups, where TLH was $(177.2 \pm 80) \mathrm{ml}$ and TLH with coagulation of uterine artery at its origin was $(154.9 \pm 30.2) \mathrm{ml}$.

But, Poojari et al. [5] study differs from our study; where the conventional TLH was $(70 \mathrm{ml})$ and TLH with Prior Uterine Artery Ligation at its origin was $(43 \mathrm{ml})$ with a statistically significant difference.

Also, the study results of Sinha et al. ${ }^{[4]}$ differ from this present study results; where the Total Laparoscopic Hysterectomy with earlier uterine ligation was $(50 \mathrm{ml})$ and 2nd group classical Total Laparoscopic Hysterectomy was $(60 \mathrm{ml})$ with significant difference of both groups.

Kale et al. ${ }^{[6]}$ study also differs from our study; where TLH was $(109.38 \pm 33.03) \mathrm{ml}$ and TLH with Uterine Artery Ligation at the beginning of surgery was $(47.50 \pm 8.12) \mathrm{ml}$ with significant differences.

As regard to intra-operative and post-operative complications no intra or post-operative complications recorded in present study but Poojari et al. ${ }^{[5]}$ recorded one patient in group of TLH with earlier Uterine Artery Ligation at its origin suffering from numerous fibroids and prior two lower segment cesarean section experienced injury of the urinary bladder. Also, Sinha et al. ${ }^{[4]}$ study recorded one patient suffering from secondary hemorrhage and vault suture. In group of classical TLH, there were two patients suffering from blood loss and need 4 unit blood transfusions.

\section{CONCLUSION}

Total Laparoscopic Hysterectomy with Prior Uterine Artery Ligation at its origin by Hem-o-Lok clips is safe effective procedure and reducing the duration of surgery.

DISCLOSURE STATEMENT: Authors declare that they have no conflicts of interest and nothing to disclose.

SOURCE OF FUNDING: There is no specific grant from any funding agency.

\section{REFERENCES}

1. Albright BB, Witte T, Tofte AN, Chou J, Black JD, Desai VB, Erekson EA. Robotic Versus Laparoscopic Hysterectomy for Benign Disease: A Systematic Review and Meta-Analysis of Randomized Trials. J Minim Invasive Gynecol. 2016 Jan;23(1):18-27. DOI: 10.1016/j.jmig.2015.08.003.

2. Dolanbay M, Kutuk MS, Ozgun MT, Uludag S, Sahin Y. Laparoscopically-assisted vaginal hysterectomy for enlarged uterus: operative outcomes and the learning curve. Ginekol Pol. 2016;87(5):333-7. DOI: 10.5603/GP.2016.0003.

3. Chiang A, Yu S, Parker WH. Laparoscopic Hysterectomy. In: Shoupe D, editor, Handbook of Gynecology. Cham: Springer International Publishing; 201. p. 651-64. DOl: 10.1007/978-3319-17798-4_83.

4. Sinha R, Sundaram M, Nikam YA, Hegde A, Mahajan C. Total laparoscopic hysterectomy with earlier uterine artery ligation. $J$ Minim Invasive Gynecol. 2008 May-Jun;15(3):355-9. DOI: 10.1016/j.jmig.2008.01.012.

5. Poojari VG, Bhat VV, Bhat R. Total laparoscopic hysterectomy with prior uterine artery ligation at its origin. Int J Reprod Med. 2014;2014:420926. DOI: 10.1155/2014/420926.

6. Kale A, Aksu S, Terzi H, Demirayak G, Turkay U, Sendag F. Uterine artery ligation at the beginning of total laparoscopic hysterectomy reduces total blood loss and operation duration. J Obstet Gynaecol. 2015;35(6):612-5. DOI: 10.3109/01443615. 2014.990431.

7. Pan HS, Ko ML, Huang LW, Chang JZ, Hwang JL, Chen SC. Total laparoscopic hysterectomy (TLH) versus coagulation of uterine arteries (CUA) at their origin plus total laparoscopic hysterectomy (TLH) for the management of myoma and adenomyosis. Minim Invasive Ther Allied Technol. 2008;17(5):318-22. DOI: 10.1080/13645700802274588. 


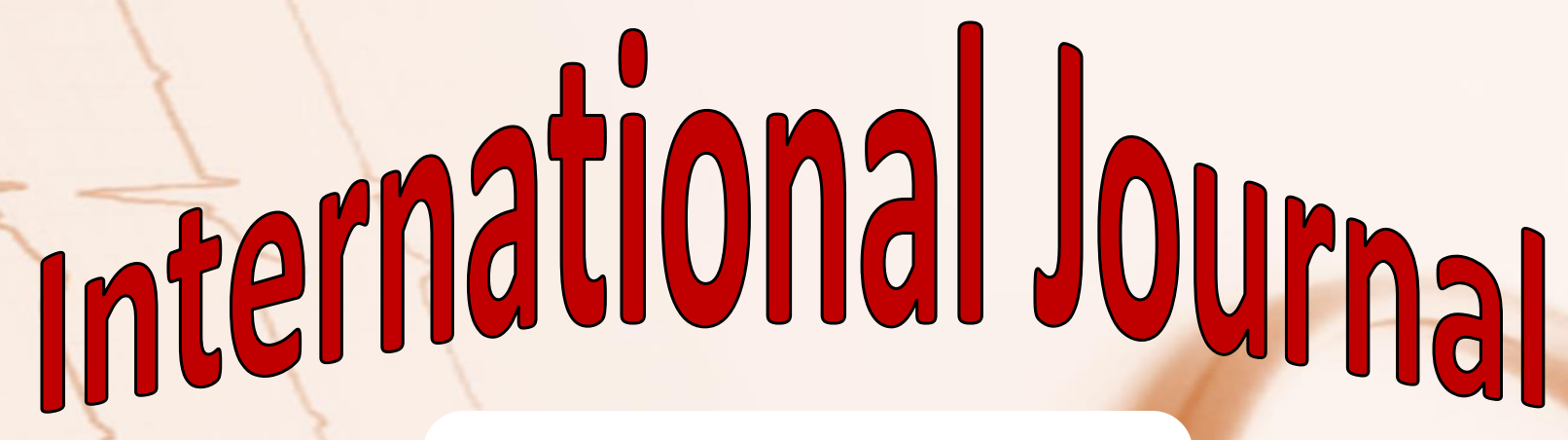

https://ijma.journals.ekb.eg/ Print ISSN: 2636-4174 Online ISSN: 2682-3780

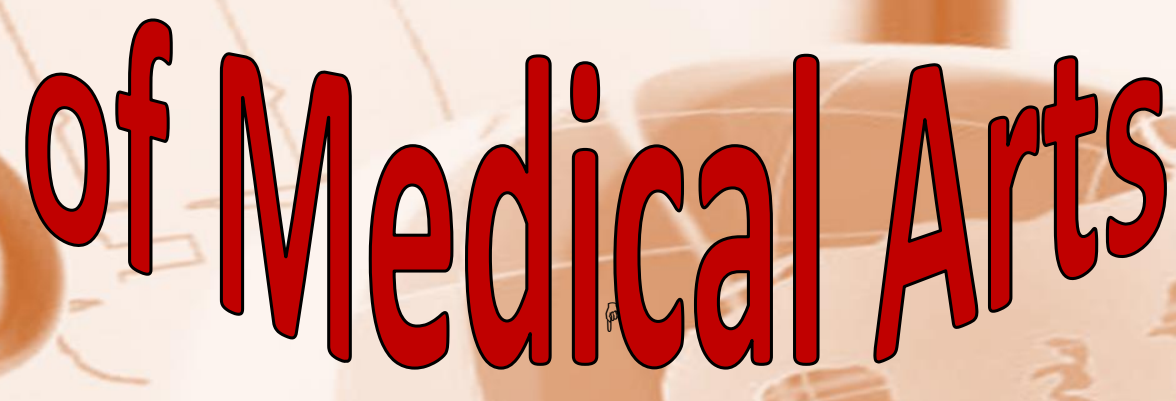

